\title{
The Extremely Efficient New Model in Milling of Complicated Surfaces in 5-Axis Machining for 3-Dimensional Contact
}

\author{
Yi Lou' ${ }^{1}$ Huran Liu', Bo Tan² \\ ${ }^{1}$ Department of Mechanical Engineering, Zhejiang University of Science and Technology, Hangzhou, China \\ ${ }^{2}$ The State Key Laboratory of Digital Manufacturing Equipment and Technology, Huazhong University of Science and \\ Technology, Wuhan, China \\ Email: liuhr53@126.com
}

How to cite this paper: Lou, Y., Liu, H.R. and Tan, B. (2018) The Extremely Efficient New Model in Milling of Complicated Surfaces in 5-Axis Machining for 3-Dimensional Contact. World Journal of Engineering and Technology, 6, 625-630.

https://doi.org/10.4236/wjet.2018.63038

Received: May 10, 2018

Accepted: July 28, 2018

Published: July 31, 2018

Copyright $\odot 2018$ by authors and Scientific Research Publishing Inc. This work is licensed under the Creative Commons Attribution International License (CC BY 4.0).

http://creativecommons.org/licenses/by/4.0/

(c) (i) Open Access

\begin{abstract}
Although most of the 5-axis NC milling machine tool producers declare that their machine poses the function of 5-axis simultaneous machining, and most of the commercialized CAD/CAM software was reported it can support the 5 -axis simultaneous machining, but there are very few analyses about their machining efficiency. I hope that the reader would be interested to know how the new method is highly effective. The paper did a good job in motivating the problem mentioned here. The model of this paper is extremely efficient ant. This is a highly effective way of surface machining. There are also some areas that need improving. The mathematic presentation is not clear, but much more pages are needed, if the authors like to make it clearer. In the resent paper "The Extreme Efficiency of the New Model in Milling of Complicated Surfaces" we discussed the extreme efficiency of the new model in milling of complicated surfaces in 5-axis machining for 2-dimensional contact. While this time let's discuss the same problem but much complicated one, 5-axis machining for 3-dimensional contact. As the research activities conducted by Dr. Liu Huran opened a new field and wide horizons in 5-axis machining of sculptured complicated surfaces using mathematical theories, and according to the interesting results presented in paper, Dr. Liu Huran is strongly encouraged if he can submit a similar work but this time with a new title that corresponds to the purpose [1].
\end{abstract}

\section{Keywords}

Surface, Contact, NC Machining

\section{Introduction}

With the rapid development of computer technology and control technology, 
five-axis NC machine plays a more and more important role in high efficiency and high precision manufacture. The small amount and high precision products provide wide application and developable perspective for 5-axis NC machine. It makes a fundamental change for NC programming [2].

This paper introduces currency method of NC machining method, develops the machining efficiency and precision of NC machine, and comes to conclusion that the local contact tool path can increase machining efficiency and sweep surface of the cutting circle on close contact path can increase machining precision [3].

The sweep surface of cutting-circle of cylinder-end milling along close contact path is calculated from relative movement. This paper compares the curvature of sweep surface and the part surface along any direction in tangent plan, discusses the condition of avoiding gouging, obtaining the optimal tool position and direction. This paper also analyzes the tolerance of five-axis NC machining along the direction of cutting and cutting strip [4].

Authors have researched the "cutter-contact" principle of differential geometry in NC machine, analyzed the condition of "close contact" in NC machining and the condition of three-close-contact in local coordinate system, and used the figure of section analyzing the degree of contact between cutting circle and surface [5].

Method how to simulate the machining process in AutoCAD is presented in this thesis, which analyzes the generation of 3D solid, obtains the geometry and topology data of solid boundary surface. This paper uses the NURB surface describing surface model, uses Boolean operation to simulate the machining process with the file of tool position and direction angle [6] [7] [8] [9].

Explanation to the Extreme Efficiency of New NC Method: the thinking Route [1]:

a) The local development or the local reconfiguration of the surface on any one point;

b) None vertical transformation in order to transformed into the vertical standard system;

c) The condition of best machining posture in order to get highest efficiency of NC machining;

d) The extremely efficient cutting method on the local point to be machined;

e) The steps of calculation (take an elliptic ball for example);

f) The Coordinate Transformation in order to be changed into coordinate system of the machine tool.

\section{The 2nd-Order Approximation of the Surface}

Use the Maclaulin series to develop the surface in the adjacent reign of point $M_{0}$ (Figure 1)

$$
r(u, v)=r\left(u_{0}, v_{0}\right)+\left(\frac{\partial}{\partial u} \Delta u+\frac{\partial}{\partial v} \Delta v\right) r+\frac{1}{2 !}\left(\frac{\partial}{\partial u} \Delta u+\frac{\partial}{\partial v} \Delta v\right)^{2} r+\cdots
$$




$$
\begin{aligned}
\boldsymbol{M M}_{0} & =r(u, v)-r\left(u_{0}, v_{0}\right) \\
& =\left(\frac{\partial}{\partial u} \Delta u+\frac{\partial}{\partial v} \Delta v\right) r+\frac{1}{2 !}\left(\frac{\partial}{\partial u} \Delta u+\frac{\partial}{\partial v} \Delta v\right)^{2} r+\cdots
\end{aligned}
$$

\section{The Condition of the Local Contact}

As show in Figure 2, the coordination of transformation in the local system

$$
\begin{gathered}
x=r(\cos \theta-1) \cos \lambda \cos \omega-r \sin \theta \sin \omega \\
y=r(\cos \theta-1) \cos \lambda \sin \omega+r \sin \theta \cos \omega \\
z=-r(\cos \theta-1) \sin \lambda
\end{gathered}
$$

\section{The 2nd Order Local Contact Method in 5-Axis NC Milling of Complicated Surface (Take an Ellipsoid Dir for Example)}

The surface to be machined is an ellipsoid dir.

$$
\begin{gathered}
r(u, v)=(A \cos u \cos v, B \cos u \sin v, C \sin u) \\
r_{u}=[-A \sin u \cos v,-B \sin u \sin v, C \cos u] \\
r_{v}=[-A \cos u \sin v, B \cos u \cos v, 0] \\
n(u, v)=(C B \cos u \cos v, A C \sin u \sin v, A B \cos u) / D \\
D=\sqrt{A^{2} B^{2} \cos ^{2} u \cos ^{2} v+A^{2} C^{2} \cos ^{2} u \sin ^{2} v+A^{2} B^{2} \sin ^{2} u}
\end{gathered}
$$

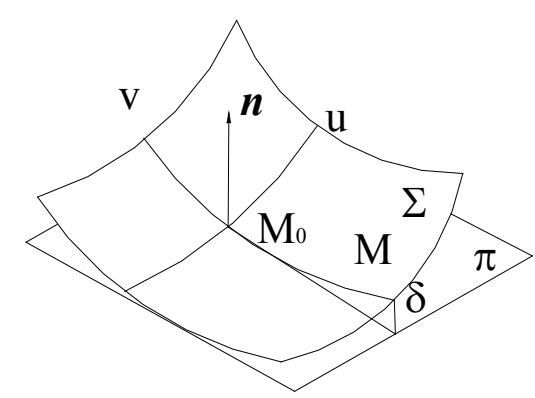

Figure 1. The distance to the tangential plan.

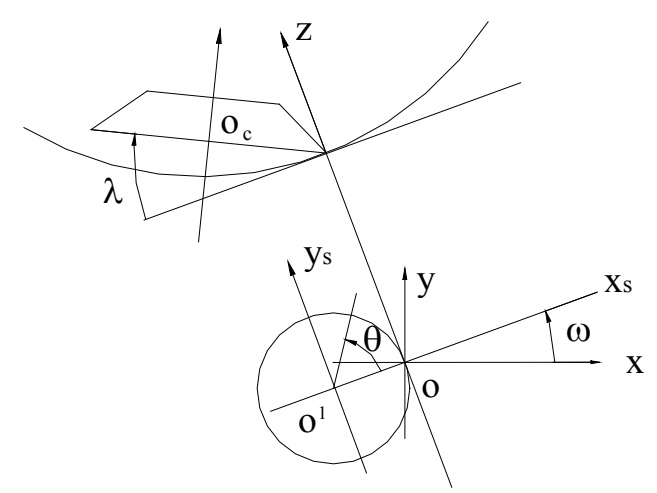

Figure 2. The position and coordination of the cutter. 


$$
\begin{gathered}
r_{u u}=[-A \cos u \cos v,-B \cos u \sin v,-C \sin u] \\
r_{u v}=[A \sin u \sin v,-B \sin u \cos v, 0] \\
r_{v v}=[-A \cos u \cos v,-B \cos u \sin v, 0] \\
E=r_{u} \cdot r_{u}=A^{2} \sin ^{2} u \cos ^{2} v+B^{2} \sin ^{2} u \sin ^{2} v+C^{2} \cos ^{2} u \\
F=0 \\
G=r_{v} \cdot r_{v}=A^{2} \cos ^{2} u \sin ^{2} v+B^{2} \cos ^{2} u \cos ^{2} v \\
n \cdot r_{u u}=\frac{A B C}{D}, a=\frac{A B C}{2 D E} \\
n \cdot r_{u v}=0, b=0 \\
n \cdot r_{v v}=\frac{A B C}{D} \cos ^{2} u, c=\frac{A B C}{2 D G} \cos ^{2} u
\end{gathered}
$$

\section{The Coordinate Transformation from Local System to the System of Machine Tool}

The orientation of the cutter center at the local system

$$
q=(\sin \lambda, 0, \cos \lambda)=(0.13,0,0.99)
$$

the coordinates of the surface:

$$
\begin{aligned}
\boldsymbol{e}_{u}= & \frac{r_{u}}{\left|r_{u}\right|} \\
= & \frac{1}{\sqrt{E}}(\boldsymbol{i} A \cos u, \boldsymbol{j} B \sin u \cos v,-\boldsymbol{k} C \sin u \sin v) \\
= & \frac{1}{\sqrt{14356.200}}(\boldsymbol{i} \times 120 \times 0.985, \boldsymbol{j} \times 40 \times 0.174 \times 0.174,-\boldsymbol{k} \times 20 \times 0.174 \times(-0.985)) \\
= & 0.986 \boldsymbol{i}-0.06 \boldsymbol{j}+0.03 \boldsymbol{k} \\
& \boldsymbol{e}_{v}=\frac{r_{v}}{\left|r_{v}\right|}=\frac{1}{\sqrt{G}}(0,-\boldsymbol{j} B \cos u \sin v, \boldsymbol{k} C \cos u \cos v) \\
& =\frac{1}{\sqrt{1517.9}}(0,-\boldsymbol{j} \times 40 \times 0.985 \times(-0.985), \boldsymbol{k} \times 20 \times 0.985 \times 0.174)
\end{aligned}
$$

\section{Computer Aided Simulation (Figure 3 and Figure 4)}

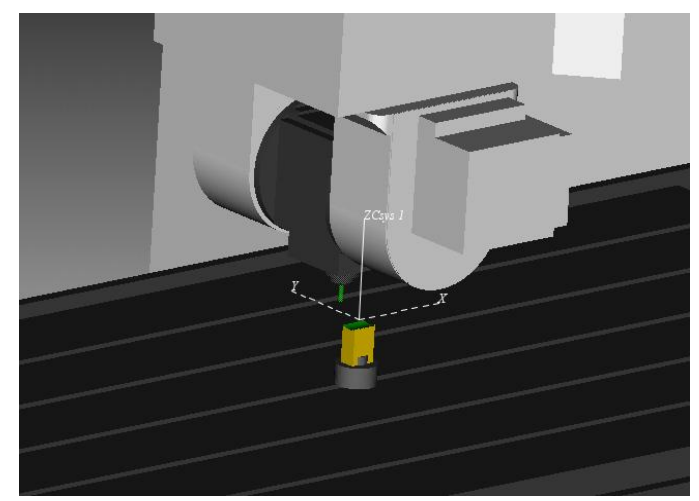

Figure 3. The machine tool. 


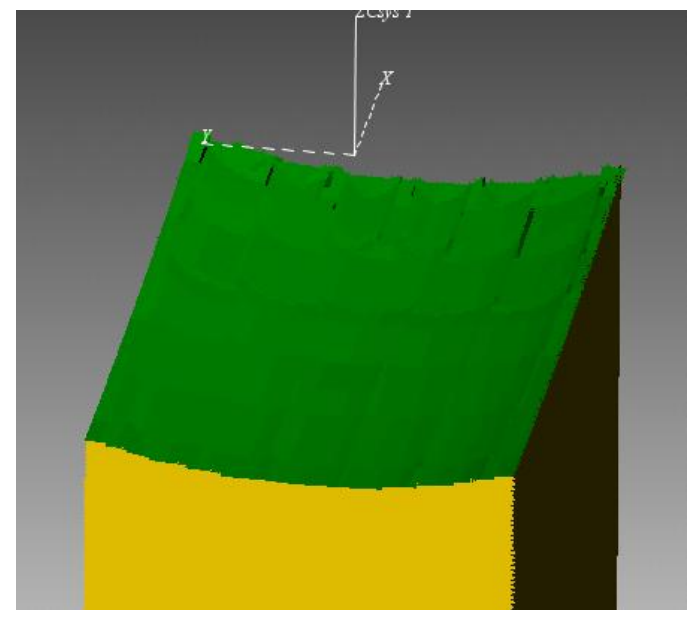

Figure 4. The machined surface.

\section{Conclusion}

5-axis machining is difficult, in ordinary factory; the NC programming is automatically generated by the software; the efficiency is unknown. In this paper, a machining theory is presented. The general machining program and the gap between tool and the work piece are a large interval. While in this paper, the cutter and the surface are contacted closely, so the machining efficiency is high.

\section{Acknowledgements}

Supported by the State Key Laboratory of Digital Manufacturing Equipment and Technology of China, Huazhong University of Science and Technology: DMETKF2015016.

Supported by the State Key Laboratory of Tribology of China, Tsinghua University: SKLTK14A06.

\section{Conflicts of Interest}

The authors declare no conflicts of interest regarding the publication of this paper.

\section{References}

[1] Liu, H.R. (2013) The Extreme Efficiency of the New Model in Milling of Complicated Surfaces. International Journal of Advanced Manufacture Technology, 67, 2765-2770.

[2] Susan, X.L. (1994) 5-Axis Machining of Sculptured Surfaces with a Flat-End Cutter. Computer-Aided Design, 26, 165-178. https://doi.org/10.1016/0010-4485(94)90040-X

[3] Lee, Y.-S. (1998) Non-Isoparametric Tool Path Planning by Machining Strip Evaluation for 5-Axis Sculptured Surface Machining. Computer-Aided Design, 30, 559-570.

[4] Lee, Y.-S. (1997) Admissible Tool Orientation Control of Gouging Avoidance for 5-Axis Complex Surface. Aided Design, 29, 507-521. 
https://doi.org/10.1016/S0010-4485(97)00002-X

[5] Vhoi, B.K. (1993) Cutter-Location Data Optimization in 5-Axis Surface Machining. Computer-Aided Design, 25, 377-386.

https://doi.org/10.1016/0010-4485(93)90033-K

[6] Kim, B.H. (1994) Effect of Cutter Mark on Surface Roughness and Scallop Height in Sculptured Surface. Computer-Aided Design, 2, 179-188. https://doi.org/10.1016/0010-4485(94)90041-8

[7] Liu, X.-W. (1995) Five-Axis NC Cylindrical Milling of Sculptured Surfaces. Computer-Aided Design, 27, 887-894. https://doi.org/10.1016/0010-4485(95)00005-4

[8] Hwang, J.S. (1992) Interference-Free Tool-Path Generation in the NC Machining of Parametric Compound Surfaces. Computer-Aided Design, 24, 667-676. https://doi.org/10.1016/0010-4485(92)90022-3

[9] Lo, C.-C. (2000)CNC Machine Tool Surface Interpolator for Ball-End Milling of Free-Form Surfaces. International Journal of Machine Tool \& Manufacture, 40, 307-326. https://doi.org/10.1016/S0890-6955(99)00071-1 